\title{
THE GROUP GENERATED BY UNIPOTENT OPERATORS ${ }^{1}$
}

\author{
C. K. FONG AND A. R. SOUROUR
}

\begin{abstract}
The group generated by unipotent $n \times n$ complex matrices is $\mathrm{SL}_{n}(C)$, and every member of the latter is a product of three unipotent matrices. The group generated by unipotent operators on Hilbert space $\mathscr{H}$ is $\mathrm{GL}(\mathscr{H})$, and every invertible operator is a product of six unipotent operators of order 2.
\end{abstract}

An operator $U$ on a Hilbert space is called unipotent [3, p. 100] if $U=1+N$, where $N$ is nilpotent. It is called unipotent of order $n$ if $N^{n}=0$ and $N^{n-1} \neq 0$. Our aim is to characterize the group $\mathscr{G}$ generated by the unipotent operators and to show that every element of $\mathscr{G}$ is a product of a small number of unipotents. In a finite-dimensional space it is obvious that every operator in $\mathscr{G}$ has determinant one. We prove the converse of this. More precisely, we show that every operator with determinant 1 is a product of three unipotents. In the infinite-dimensional case we show that $\mathscr{G}$ is the group of all invertible operators and that every operator in $\mathscr{G}$ is a product of six unipotents of order 2 .

We point out that other generators for the group of invertible operators were obtained by Radjavi [4].

The finite-dimensional case. In what follows $\mathscr{V}$ is an $n$-dimensional vector space over the complex field, where $n$ is finite. The algebra of all linear transformations on $\mathscr{V}$ is denoted by $L(\mathscr{V})$. A linear transformation $T$ on $\mathscr{V}$ is called cyclic if there exists a vector $x$ such that the vectors $x, T x, T^{2} x, \ldots, T^{n-1} x$ span $\mathscr{V}$. It is well known that $T$ is cyclic if and only if its Jordan canonical form contains only one Jordan block for every eigenvalue, and that this is the case if and only if the minimal polynomial of $T$ equals its characteristic polynomial (see [2, Chapter 7]). As usual, the group of $n \times n$ complex matrices with determinant 1 is denoted by $\operatorname{SL}_{n}(C)$.

LEMMA 1. For each invertible $A \in L(\mathscr{V})$ with a singleton spectrum, there exists a unipotent $U$ such that $U A$ is cyclic and $\sigma(U A)=\sigma(A)$.

Proof. Let $\sigma(A)=\{\lambda\}$, and let $N=A-\lambda 1$. By considering the Jordan canonical form, we see that there exists a basis $\left\{e_{1}, e_{2}, \ldots, e_{n}\right\}$ of $\mathscr{V}$ such that $N e_{j}=\varepsilon_{j} e_{j+1}$ $(1 \leqslant j \leqslant n-1)$ and $N e_{n}=0$, where $\varepsilon_{j}=0$ or 1 . Let $S$ be the usual shift; i.e., $S e_{j}=e_{j+1}(1 \leqslant j \leqslant n-1)$ and $S e_{n}=0$, and then let $J=S-N$. If $U=1+\lambda^{-1} J$,

Received by the editors December 19, 1984 and, in revised form, April 23, 1985.

1980 Mathematics Subject Classification. Primary 47B99, 47D10; Secondary 15A30.

${ }^{1}$ This research has been supported by NSERC grants U0072 and A3674. 
then $U$ is unipotent and $U A=\lambda 1+S-S^{2} D$ for some transformation $D$ which is diagonal with respect to the basis under consideration. Using the fact that $D S=$ $S S^{*} D S$ and using induction, we get that for every positive integer $m,(U A-\lambda 1)^{m}$ $=S^{m}+S^{m+1} R_{m}$ for some $R_{m} \in L(\mathscr{V})$. In particular, $(U A-\lambda 1)^{n-1} \neq 0$, but $(U A-\lambda 1)^{n}=0$. This shows that $\sigma(U A)=\{\lambda\}$ and that $U A$ is cyclic.

LEMMA 2. For each invertible $A \in L(\mathscr{V})$, there exists a unipotent $U$ such that $U A$ is cyclic.

Proof. We may write $A$ as a direct sum $A=A_{1} \oplus \cdots \oplus A_{k}$, where $\sigma\left(A_{j}\right)=\left\{\lambda_{j}\right\}$ and $\lambda_{i} \neq \lambda_{j}$ for $i \neq j$. By Lemma 1 , there are unipotents $U_{j}$ such that $U_{j} A_{j}$ is cyclic and $\sigma\left(U_{j} A_{j}\right)=\left\{\lambda_{j}\right\}$ for every $j$. If $U=U_{1} \oplus \cdots \oplus U_{k}$, then $U A$ is cyclic, since it is the direct sum of cyclic operators with disjoint spectra.

The following theorem may be of independent interest.

THEOREM 1. Let $A$ be a cyclic linear transformation, and let $\beta_{i}$ and $\gamma_{i}(1 \leqslant i \leqslant n)$ be complex numbers such that $\operatorname{det} A \prod_{i=1}^{n} \beta_{i}=\prod_{i=1}^{n} \gamma_{i}$. Then there exists a linear transformation $B$ such that $B$ has eigenvalues $\beta_{1}, \ldots, \beta_{n}$ and $B A$ has eigenvalues $\gamma_{1}, \ldots, \gamma_{n}$. The eigenvalues are repeated according to algebraic multiplicity.

Proof. Let $f(x)=a_{0}+a_{1} x+\cdots+a_{n-1} x^{n-1}+x^{n}$ be the characteristic polynomial of $A$. By taking an appropriate basis, we may assume that the matrix of $A$ is the companion matrix of $f(x)$-i.e., the matrix

$$
C=\left[\begin{array}{ccccc}
0 & 0 & 0 & \cdots & -a_{0} \\
1 & 0 & 0 & \cdots & -a_{1} \\
0 & 1 & 0 & \cdots & -a_{2} \\
& & & & \vdots \\
0 & 0 & \cdots & 1 & -a_{n-1}
\end{array}\right] .
$$

We will show that there exist complex numbers $t_{1}, t_{2}, \ldots, t_{n-1}$ such that if

$$
D=\left[\begin{array}{lllll}
\beta_{1} & t_{1} & 0 & \cdots & 0 \\
0 & \beta_{2} & t_{2} & \cdots & 0 \\
& & & & \vdots \\
& & & & t_{n-1} \\
0 & 0 & \cdots & 0 & \beta_{n}
\end{array}\right],
$$

then $D C$ has eigenvalues $\gamma_{1}, \gamma_{2}, \ldots, \gamma_{n}$.

The proof is by induction on $n$. There is nothing to prove for $n=1$. Now suppose the above is true for $(n-1) \times(n-1)$ matrices $(n \geqslant 2)$, and let $C$ be as above. Let $g(x)=\left(x-\gamma_{1}\right) \cdots\left(x-\gamma_{n}\right)$. We can write $g(x)$ in the form $g(0)+x g_{1}(x)$ for a monic polynomial $g_{1}(x)$ of degree $n-1$. Let $t_{1}$ be a root of the polynomial equation $g_{1}(x)-a_{1} \beta_{2} \cdots \beta_{n}=0$. It follows that $t_{1}$ satisfies the equation $x g_{1}(x)-$ $t_{1} a_{1} \beta_{2} \cdots \beta_{n}=0$, and so there exists a monic polynomial $h(x)$ of degree $n-1$ 
such that

$$
x g_{1}(x)-t_{1} a_{1} \beta_{2} \cdots \beta_{n}=\left(x-t_{1}\right) h(x)
$$

It follows that $h(0)=a_{1} \beta_{2} \cdots \beta_{n}$, and so the product of the zeros of $h(x)$ is $(-1)^{n-1} a_{1} \beta_{2} \cdots \beta_{n}$. By the induction hypothesis, there exist complex numbers $t_{2}, \ldots, t_{n-1}$ such that the characteristic polynomial of $D_{0} C_{0}$ is $h(x)$, where $C_{0}$ is the companion matrix of the polynomial $a_{1}+a_{2} x+\cdots+a_{n-1} x^{n-1}+x^{n}$; and where $D_{0}$ is the matrix with $\beta_{2}, \ldots, \beta_{n}$ on the main diagonal, $t_{2}, \ldots, t_{n-1}$ on the diagonal above the main one, and zero everywhere else. Now let $D$ be the $n \times n$ matrix with main diagonal $\beta_{1}, \ldots, \beta_{n}$, with $t_{1}, \ldots, t_{n-1}$ on the diagonal above, and with other entries zero. We have

$$
D C=\left[\begin{array}{c|cc}
t_{1} & 0 \cdots 0 & \cdots \\
\hline \beta_{2} & \\
0 & \\
\vdots & D_{0} C_{0} \\
0 &
\end{array}\right]
$$

where $\mu=-\left(a_{0} \beta_{1}+a_{1} t_{1}\right)$. By direct computation, we see that the characteristic polynomial of $D C$ is

$$
\begin{gathered}
\left(x-t_{1}\right) \operatorname{det}\left(x I-D_{0} C_{0}\right)-\mu \beta_{2} \cdots \beta_{n} \\
=\left(x-t_{1}\right) h(x)+a_{0} \prod_{i=1}^{n} \beta_{i}+t_{1} a_{1} \prod_{i=2}^{n} \beta_{i} \\
=x g_{1}(x)+a_{0} \beta_{1} \beta_{2} \cdots \beta_{n}=g(x),
\end{gathered}
$$

so the eigenvalues of $D C$ are $\gamma_{1}, \ldots, \gamma_{n}$. This ends the induction proof.

The previous theorem implies that every cyclic $A$ with determinant 1 is a product of two unipotents. Combining this with Lemmas 1 and 2, we have the following theorem.

THEOREM 2. Every linear transformation with determinant 1 is a product of three unipotent transformations.

The infinite-dimensional case. We consider a separable infinite-dimensional Hilbert space $\mathscr{H}$ over the complex field. The algebra of all bounded linear operators on $\mathscr{H}$ is denoted by $B(\mathscr{H})$, and the group of all invertible operators is denoted by GL( $\mathscr{H})$. Let $\mathscr{U}$ be the set of all unipotent operators of order 2 . The set of all products of $n$ operators in $\mathscr{U}$ is denoted by $\mathscr{U}^{n}$. For an operator $T$, an equation such as $T=\left(\begin{array}{ll}A & B \\ C & D\end{array}\right)$ means that there is a unitary operator from $\mathscr{H}$ to $\mathscr{H} \oplus \mathscr{H}$ which carries $T$ to the operator $\left(\begin{array}{ll}A & B \\ C & D\end{array}\right)$ on $\mathscr{H} \oplus \mathscr{H}$.

We start with two lemmas.

Lemma 3. If both $A$ and $1+A$ are invertible, then $A \oplus A^{-1} \in \mathscr{U}^{2}$. 
Proof. Let

$$
\begin{aligned}
& F_{1}(A)=\left(\begin{array}{ll}
2 A(1+A)^{-1} & (A-1)(1+A)^{-1} \\
(1-A)(1+A)^{-1} & 2(1+A)^{-1}
\end{array}\right), \\
& F_{2}(A)=\left(\begin{array}{ll}
2 A(1+A)^{-1} & \left(A^{-1}-1\right)(1+A)^{-1} \\
\left(A^{2}-A\right)(1+A)^{-1} & 2(1+A)^{-1}
\end{array}\right) .
\end{aligned}
$$

Then both $F_{1}(A), F_{2}(A)$ are in $\mathscr{U}$, and $A \oplus A^{-1}=F_{1}(A) F_{2}(A)$.

LeMMA 4. If $|\alpha|=1$ and $C$ is invertible, then $\alpha 1 \oplus C \in \mathscr{U}^{4}$.

Proof. Choose $\varepsilon>0$ such that $\varepsilon<1$ and $\varepsilon\|C\|<1$. Write $D=\varepsilon C$. Now $\alpha 1 \oplus C$ is unitarily equivalent to

$$
\cdots \oplus \alpha 1 \oplus \alpha 1 \oplus C \oplus \alpha 1 \oplus \alpha 1 \oplus \cdots,
$$

which is the product of the following two operators:

$$
\begin{aligned}
& A=\cdots \oplus \alpha^{4} D \oplus \bar{\alpha}^{2} D^{-1} \oplus \alpha^{2} D \oplus D^{-1} \oplus D \oplus \alpha \varepsilon^{-1} 1 \oplus \bar{\alpha} \varepsilon 1 \oplus \alpha^{3} \varepsilon^{-1} 1 \oplus \cdots \\
& B=\cdots \oplus \bar{\alpha}^{3} D^{-1} \oplus \alpha^{3} D \oplus \bar{\alpha} D^{-1} \oplus \alpha D \oplus \varepsilon^{-1} 1 \oplus \varepsilon 1 \oplus \alpha^{2} \varepsilon^{-1} 1 \oplus \bar{\alpha}^{2} \varepsilon 1 \oplus \cdots
\end{aligned}
$$

Following the proof of Lemma 3, we have

$$
\begin{gathered}
\alpha^{n} D \oplus \alpha^{-n} D^{-1}=F_{1}\left(\alpha^{n} D\right) F_{2}\left(\alpha^{n} D\right), \\
\bar{\alpha}^{n} \varepsilon 1 \oplus \alpha^{n} \varepsilon^{-1} 1=F_{1}\left(\bar{\alpha}^{n} \varepsilon 1\right) F_{2}\left(\bar{\alpha}^{n} \varepsilon 1\right) .
\end{gathered}
$$

Notice that $\sup _{n}\left\|F_{1}\left(\alpha^{n} D\right)\right\|<\infty, \sup _{n}\left\|F_{2}\left(\alpha^{n} D\right)\right\|<\infty, \sup _{n}\left\|F_{1}\left(\bar{\alpha}^{n} \varepsilon 1\right)\right\|<\infty$ and $\sup _{n}\left\|F_{2}\left(\bar{\alpha}^{n} \varepsilon 1\right)\right\|<\infty$. Thus $A$ is unitarily equivalent to the product $A_{1} A_{2}$, where

$$
A_{j}=\cdots \oplus F_{j}\left(\alpha^{4} D\right) \oplus F_{j}\left(\alpha^{2} D\right) \oplus F_{j}(D) \oplus F_{j}(\bar{\alpha} \varepsilon 1) \oplus F_{j}\left(\bar{\alpha}^{3} \varepsilon 1\right) \oplus \cdots
$$

$(j=1,2)$. It follows from the proof of Lemma 1 that both $A_{1}$ and $A_{2}$ are in $\mathscr{U}$, and hence $A \in \mathscr{U}^{2}$. In the same way we have $B \in \mathscr{U}^{2}$. Therefore $\alpha I \oplus C \in \mathscr{U}^{4}$.

THEOREM 3. GL( $\mathscr{H})=\mathscr{U}^{6}$.

Proof. We consider two cases. In the first case, we assume that $T$ is not a scalar modulo the compacts. By a theorem of Brown and Pearcy [1, Corollary 3.4], $T$ is similar to an operator of the form $\left(\begin{array}{c}* B \\ *\end{array}\right)$. Notice that

$$
\left(\begin{array}{rr}
1 & -B \\
0 & 1
\end{array}\right)\left(\begin{array}{ll}
* & B \\
* & 1
\end{array}\right)=\left(\begin{array}{ll}
A & 0 \\
C & 1
\end{array}\right),
$$

where $A$ is some invertible operator. (That $A$ is invertible follows from the fact that $T$ is invertible.) Also,

$$
\left(\begin{array}{ll}
1 & 0 \\
X & 1
\end{array}\right)\left(\begin{array}{ll}
A & 0 \\
C & 1
\end{array}\right)=\left(\begin{array}{ll}
A & 0 \\
0 & 1
\end{array}\right)
$$

where $X=-C A^{-1}$. By Lemma $4,\left(\begin{array}{ll}A & 0 \\ 0 & 1\end{array}\right) \in \mathscr{U}^{4}$. Since $T$ is similar to

we have $T \in \mathscr{U}^{6}$.

$$
\left(\begin{array}{ll}
1 & B \\
0 & 1
\end{array}\right)\left(\begin{array}{rr}
1 & 0 \\
-X & 1
\end{array}\right)\left(\begin{array}{ll}
A & 0 \\
0 & 1
\end{array}\right)
$$


It now remains to consider the case $T=\lambda 1+K$, where $K$ is compact. We notice that $\lambda \neq 0$, since $T$ is invertible. Let $\varepsilon$ be a positive real number to be determined later. By Riesz theory, $K$ is similar to $K_{0} \oplus F$, where $K_{0}$ has spectral radius $<\varepsilon$, and where $F$ is an operator on a finite-dimensional space. By a result of Rota [6, Theorem 2], $K_{0}$ is similar to an operator whose norm is $<\varepsilon$, so we may assume without loss of generality that $\left\|K_{0}\right\|<\varepsilon$. Write

$$
K_{0}=\left(\begin{array}{ll}
K_{1} & K_{2} \\
K_{3} & K_{4}
\end{array}\right) \text {, }
$$

and let $A=\left(\lambda+K_{1}+K_{2}\right)^{-1}\left(\alpha-\lambda-K_{1}\right)$, where $\alpha=1$ if $\lambda \neq \pm 1$ and $\alpha=i$ if $\lambda= \pm 1$. (The operator $\lambda+K_{1}+K_{2}$ is invertible if $\varepsilon$ is small enough.) Let

$$
S=\left(\begin{array}{cc}
1+A & -A \\
A & 1-A
\end{array}\right)
$$

We have $(S-1)^{2}=1$. Furthermore,

$$
\left(\lambda+K_{0}\right) S=\left(\begin{array}{cc}
\alpha & \lambda-\alpha+L_{1} \\
\alpha-\lambda+L_{2} & 2 \lambda-\alpha+L_{3}
\end{array}\right),
$$

where

$$
L_{1}=K_{1}+K_{2}, \quad L_{2}=\left(K_{3}-K_{1}\right)(1+A)+\left(K_{4}-K_{2}\right) A
$$

and

$$
L_{3}=K_{1}+K_{4}+\left(K_{1}+K_{2}-K_{3}-K_{4}\right) A \text {. }
$$

Let

$$
R=\left(\begin{array}{cc}
1 & 0 \\
\bar{\alpha} \lambda-1-\bar{\alpha} L_{2} & 1
\end{array}\right)
$$

Therefore

$$
R\left(\lambda+K_{0}\right) S=\left(\begin{array}{cc}
\alpha & * \\
0 & \bar{\alpha} \lambda^{2}+J
\end{array}\right),
$$

where

$$
J=(1-\lambda \bar{\alpha})\left(L_{2}-L_{1}\right)+L_{3}-\bar{\alpha} L_{1} L_{2} .
$$

We notice that by our choice of $\alpha$ we have $\alpha \neq \bar{\alpha} \lambda^{2}$. From the expressions for $J$, $L_{1}, L_{2}, L_{3}$ we see that $J$ is compact, and that we can choose $\varepsilon$ small enough so that $\|J\| \leqslant\left|\alpha-\bar{\alpha} \lambda^{2}\right|$; so $\alpha \notin \sigma\left(\bar{\alpha} \lambda^{2}+J\right)$. Now we have

$$
R\left(\lambda+K_{0}\right) S=\left(\begin{array}{ll}
\alpha & * \\
0 & C
\end{array}\right) \quad \text { with } \alpha \notin \sigma(C) .
$$

It follows by [5, Corollary 0.15$]$ that $R\left(\lambda+K_{0}\right) S$ is similar to $\alpha 1 \oplus C$. Since

$$
(R \oplus 1)\left[\left(\lambda+K_{0}\right) \oplus(\lambda+F)\right](S \oplus 1)=R\left(\lambda+K_{0}\right) S \oplus(\lambda+F),
$$

and since $T$ is similar to $\left(\lambda+K_{0}\right) \oplus(\lambda+F)$, we have $(R \oplus 1) T(S \oplus 1)$, similar to $\alpha 1 \oplus C \oplus(\lambda+F)$, which, by Lemma 4, belongs to $\mathscr{U}^{4}$. Since $R \oplus 1$ and $S \oplus 1$ are unipotent, we get $T \in \mathscr{U}^{6}$. This ends the proof of the theorem. 


\section{REFERENCES}

1. A. Brown and C. Pearcy, Structure of commutators of operators, Ann. of Math. (2) 82 (1965), $112-127$.

2. K. Hoffman and R. Kunze, Linear algebra, 2nd ed., Prentice-Hall, Englewood Cliffs, N.J., 1971.

3. I. Kaplansky, Fields and rings, University of Chicago Press, Chicago, Ill., 1972.

4. H. Radjavi, The group generated by involutions, Proc. Roy. Irish Acad. Sect. A 81 (1981), 9-12.

5. H. Radjavi and P. Rosenthal, Invariant subspaces, Springer-Verlag, Berlin, 1973.

6. G.-C. Rota, On models for linear operators, Comm. Pure Appl. Math. 13 (1960), 469-472.

Department of Mathematics, University of Toronto, Toronto, Ontario M5S 1A1, Canada

Department of Mathematics, University of Victoria, Victoria, British Columbia V8W 2Y2, CANADA (Current address A. R. Sourour)

Current address (C. K. Fong): Department of Mathematics, University of Ottawa, Ottawa, Ontario K1N 9B4, Canada 